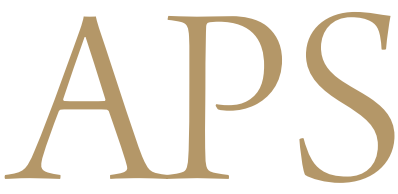

Archives of Plastic Surgery

\title{
Wound Healing Effects of Rose Placenta in a Mouse Model of Full-Thickness Wounds
}

\author{
Yang Woo Kim ${ }^{1}$, Seung Ryeol Baek ${ }^{1}$, Eun Sook Lee ${ }^{1}$, Sang Ho Lee ${ }^{2}$, Sang Hyun Moh ${ }^{3}$, \\ Soo Yun $\mathrm{Kim}^{3}$, Ji Hong $\mathrm{Moh}^{3}$, Chieko Kondo ${ }^{4}$, Young Woo Cheon ${ }^{1}$ \\ Departments of ${ }^{1}$ Plastic and Reconstructive Surgery and ${ }^{2}$ Pathology, Gil Medical Center, Gachon University School of Medicine, Incheon; \\ ${ }^{3}$ Anti-Aging Research Institute, Bio-FD\&C Co., LTD, Incheon, Korea; ${ }^{4}$ Ginza Tomato, Tokyo, Japan
}

Background Rosa damascena, a type of herb, has been used for wound healing in Eastern folk medicine. The goal of this study was to evaluate the effectiveness of rose placenta from R. damascena in a full-thickness wound model in mice.

Methods Sixty six-week-old C57BL/6N mice were used. Full-thickness wounds were made with an 8-mm diameter punch. Two wounds were made on each side of the back, and wounds were assigned randomly to the control and experimental groups. Rose placenta $(250 \mu \mathrm{g})$ was injected in the experimental group, and normal saline was injected in the control group. Wound sizes were measured with digital photography, and specimens were harvested. Immunohistochemical staining was performed to assess the expression of epidermal growth factor (EGF), vascular endothelial growth factor (VEGF), transforming growth factor- $\beta 1$ (TGF- $\beta 1$ ), and CD31. Vessel density was measured. Quantitative analysis using an enzymelinked immunosorbent assay (ELISA) for EGF was performed. All evaluations were performed on postoperative days $0,2,4,7$, and 10 . Statistical analyses were performed using the paired t-test.

Results On days 4, 7, and 10, the wounds treated with rose placenta were significantly smaller. On day 2, VEGF and EGF expression increased in the experimental group. On days 7 and 10, TGF- $\beta 1$ expression decreased in the experimental group. On day 10, vessel density increased in the experimental group. The increase in EGF on day 2 was confirmed with ELISA.

Conclusions Rose placenta was found to be associated with improved wound healing in a mouse full-thickness wound model via increased EGF release. Rose placenta may potentially be a novel drug candidate for enhancing wound healing.

Keywords Wound healing / Rosa / Epidermal growth factor / Mice
Correspondence: Young Woo Cheon Department of Plastic and Reconstructive Surgery, Gil Medical Center, Gachon University School of Medicine, 21 Namdong-daero 774beon-gil, Namdong-gu, Incheon 21565, Korea

Tel: +82-1577-2299

Fax: +82-32-461-2774

E-mail:youngwooc@gmail.com

\begin{abstract}
This research was supported by the Basic Science Research Program through the National Research Foundation of Korean (NRF), funded by the Ministry of Science, ICT \& Future Planning (NRF-2014R1A1A 1037258) and a research-focused grant from Gil Hospital (FRD 2013-41). This research was also supported by research foundation of Gachon university (GCU2013-M055).
\end{abstract}

No potential conflict of interest relevant to this article was reported.

\section{INTRODUCTION}

The incidence of chronic wounds is increasing as a consequence of increased life expectancy and the increased prevalence of chronic diseases such as diabetes mellitus and hypertension [1].
The presence of chronic wounds such as diabetic or ischemic ulcers leads to a diminished quality of life. Wound healing is a complicated process associated with various growth and environmental factors. The detailed mechanism of wound healing is still under investigation, and a continually increasing amount of 
research on wound healing has been published. Various medications using growth factors and newly developed dressing materials have been introduced. However, most of the latest medications and dressing materials are expensive or are not cost-effective. Over the years, various natural substances have been evaluated for their effects on wound healing in experimental animal models. The development of new medications or dressing materials using natural substances can promote successful wound healing at a low cost. Several plants and herbs have been empirically used as traditional medicines to treat skin disorders and injuries.

Rosa damascena is a plant in the family Rosaceae. Rosaceae members are well-known ornamental plants and roses have been referred to as the king of flowers. Historically, roses were cultivated and used for medical purposes by the ancient Chinese and Egyptians nearly 5,000 years ago, as well as in ancient Greece and the Roman Empire [2]. Several compounds, including terpenes, glycosides, flavonoids, and anthocyanins, can be isolated from roses [3]. The therapeutic uses of $R$. damascena in ancient medicine include the treatment of abdominal and chest pain, menstrual bleeding, and digestive problems; the reduction of inflammation, coughing, and thirst; and wound healing [3]. While $R$. damascena has been used as rootstock for ornamental roses in Western cultures, all parts of $R$. damascena are used in folk medicine in Eastern cultures. Specifically, rose hips have been widely used as diuretics and are registered in the Korean and Japanese Pharmacopoeia [4]. Various products and constituents isolated from the flowers, petals, and hips of this plant have been studied in vivo and in vitro, and have been shown to have antihypnotic, anticonvulsive, anti-Human immunodeficiency Virus, antidiabetic, antibacterial, and anti-inflammatory effects [5].

To date, most research on wound healing has focused on growth factors, and several growth factors have been identified as potential mediators of wound healing. Platelet-derived growth factor (PDGF), fibroblast growth factor (FGF), transforming growth factor (TGF), epidermal growth factor (EGF), and vascular endothelial growth factor (VEGF) have been reported to accelerate the formation of various components involved in the wound healing process [6]. PDGF and FGF have been found to accelerate the rate of granulation tissue formation and wound closure in diabetic mice [7], while TGF has been shown to improve the healing of partial-thickness wounds in pigs [8]. EGF is the predominant growth factor involved in the epithelialization of skin wounds in vivo and functions by accelerating keratinocyte migration [9]. VEGF accelerates wound closure by mediating angiogenic activity during the proliferative phase of wound healing [10].
However, directly applying growth factors to wounds has several limitations. Growth factors have a short half-life, are difficult to deliver to the target site, and are expensive to manufacture.

We therefore searched for natural and inexpensive agents that can initiate the release of growth factors. $R$. damascena was identified as a possible candidate. This study focused on the effects of rose placenta isolated from $R$. damascena on the wound healing process and attempted to characterize the mechanisms through which it exerted its effects.

\section{METHODS}

Preparation of rose placenta extract from $R$. damascena Rose placenta was prepared using cultured rose placental cells. Briefly, hot-air-dried callus cells ( $0.2 \mathrm{~g})$ collected from rose placenta were mixed with $50 \%$ ethanol $(100 \mathrm{~mL})$ and sonicated $\left(70^{\circ} \mathrm{C}, 100\right.$ minutes). After sonication, the extracted specimen $(50 \mathrm{~mL})$ was centrifuged $(9,000 \mathrm{rpm}$, five minutes) and the supernatant was collected. The supernatant was filtered through a $0.45-\mu \mathrm{m}$ membrane and freeze-dried at $-20^{\circ} \mathrm{C}$ for $12-24$ hours. The freeze-dried specimen was diluted with water to yield the rose placenta preparation. Rose placenta $(1 \mu \mathrm{g} / \mu \mathrm{L})$ was used for the wound healing experiments.

\section{Animal study}

Sixty six-week-old C57BL/6N mice (Orient Bio Inc., Seongnam, Korea) of average weight $(18-20 \mathrm{~g})$ were used. Each mouse was anesthetized with an intraperitoneal injection of $0.006 \mathrm{~mL} / 10 \mathrm{~g}$ Zolitel and $0.004 \mathrm{~mL} / 10 \mathrm{~g}$ Rompun. The hair on the back of the mouse was shaved, and a full-thickness skin defect was created using an 8-mm diameter punch. Identical wounds were made on each side of the dorsal surface symmetrically. The wounds were randomly assigned to the control and experimental groups. Rose placenta $(1 \mu \mathrm{g} / \mu \mathrm{L})$ was used in the experimental group wounds, which were injected once subcutaneously with $250 \mu \mathrm{g}$ of rose placenta at four different points along the edges and base of the wound, while the control group wounds were injected with $250 \mu \mathrm{L}$ of normal saline. The wounds were maintained for 48 hours with Tegaderm (3M Health Care, Maplewood, MN, USA) to prevent dehydration. All animal procedures were approved by the Lee Gil Ya Cancer and Diabetes Institutional Animal Care and Use Committee.

\section{Wound size evaluation}

In order to evaluate changes in wound size, digital photographs were taken two, four, seven, and 10 days after the injections (Fig. 1). The images were captured by vertically positioning the camera lens over the wounds. To minimize error from camera posi- 


\section{Fig. 1. The mouse wound healing model}

A full-thickness skin defect was made on each side of the dorsal surface using an 8-mm diameter punch. Digital photographs were taken two, four, seven, and 10 days after the injections. Left, control; right, experimental.

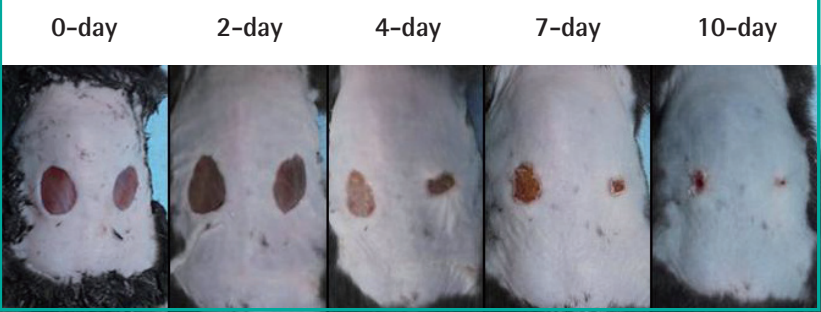

tioning, the camera was fixed at $60 \mathrm{~cm}$ apart from a subject using a stand. All images included a ruler in centimeters photographed adjacent to the wound for calibration. The digitally imaged wound was analyzed using the Image J program. The number of pixels in the area of the photograph corresponding to each wound was calculated and corrected with perspective scaling. The proportions of the wounds on days two, four, seven, and 10 were calculated in comparison to the initial defect size.

\section{Tissue collection}

The mice were anesthetized by an overdose of Zolitel and Rompun on days two, four, seven, and 10. Groups of 10 mice were sacrificed on days two, four, and seven. The remaining 30 mice were sacrificed on day 10. The whole wound was excised with a margin of approximately $2 \mathrm{~mm}$ of unwounded skin. For histopathological and immunohistological examinations, each specimen was cut in half and fixed using $10 \%$ buffered formalin solution and embedded in paraffin. Half of the tissue samples were frozen using liquid nitrogen and stored for analysis using an enzyme-linked immunosorbent assay (ELISA).

\section{Histological study}

Formalin-fixed samples were dehydrated and embedded in paraffin. The samples were sliced using a microtome $(2.5-\mu \mathrm{m}$ thick) and stained with hematoxylin and eosin for histopathological examination. Inflammatory cells were counted by two independent pathologists at a magnification of $\times 400$ in order to compare their presence in the control and experimental groups. For immunohistochemical analysis, samples were sliced and placed on slides coated with polylysine. The slides were deparaffinized on a hot plate at $60^{\circ} \mathrm{C}$ and rehydrated. The slides were completely submerged in antigen retrieval buffer and boiled for three minutes at $115^{\circ} \mathrm{C}$, then placed in phosphate-buffered saline (PBS) buffer to cool to room temperature. The slides were then washed in distilled water and again in 1\% PBS. The slides were incubated in a hydrogen peroxide solution in a humidified container for 15 minutes to eliminate endogenous peroxidase activity, washed in PBS, and incubated with Dako blocking solution (Vector/BLOXALL blocking solution, Vector lavoratories, Burlingame, CA, USA) at room temperature for 30 minutes. After incubation, the slides were washed in PBS and incubated in blocking reagent containing the diluted $(1 / 1,000)$ primary antibody (rabbit anti-TGF- $\beta 1$ antibody, Abcam, Cambridge, UK). The slides were covered with Parafilm and incubated in a humidified container for 18 hours at $4^{\circ} \mathrm{C}$. The slides were then incubated at room temperature for one hour and washed with PBS. After a brief wipe, the slides were incubated in blocking reagent containing the diluted $(1: 1,000)$ secondary antibody (goat anti-rabbit IgG, Enzo Life Sciences Inc., Farmingdale, NY, USA). The tissues were then covered with Parafilm and incubated in a humidified chamber for 30 minutes at room temperature. After incubation, the slides were treated with diaminobenzidine and counterstained with hematoxylin.

\section{Measurement of vessel density}

Capillary density was assessed by under $200 \times$ magnification by two independent pathologists who counted the number of positively stained capillaries using CD31+ immunohistochemistry. The number of capillaries was counted in 10 fields for each specimen, and the mean capillary density (number $/ \mathrm{mm}^{2}$ ) was obtained.

\section{Quantitative analysis of EGF with ELISA}

The mouse skin wounds were homogenized in RIPA buffer (10 $\mathrm{mM}$, Tris- $\mathrm{Cl}$ [pH 8.0], $1 \mathrm{mM}$ ethylene diamine tetraacetic acid, $0.5 \mathrm{mM}$ ethylene glycol tetraacetic acid, $1 \%$ Triton X-100, $0.1 \%$ sodium deoxycholate, $0.1 \%$ sodium dodecylsulfate, $140 \mathrm{mM}$ $\mathrm{NaCl}, 1 \mathrm{mM}$ PMSF) containing a mixture of protease inhibitors for 30 minutes on ice. The homogenate was centrifuged at 20,000 $\times \mathrm{g}$ for 15 minutes at $4^{\circ} \mathrm{C}$, and the supernatant were collected and measured by the bicinchoninic acid protein assay (Pierce BCA kit, Thermo Fisher Scientific, Waltham, MA, USA). EGF production was measured using a commercially available ELISA kit (PEPROTECH, Rocky Hill, NJ, USA). Briefly, sample proteins and standards were diluted, and $100 \mu \mathrm{L}$ of the diluted samples were added to each well in duplicate. The plates were covered and incubated for two hours at room temperature. The plates were washed before the addition of the detection antibody solution and incubated for two hours at room temperature. The plates were washed and incubated with streptavidinhorseradish peroxidase conjugate solution for 30 minutes. The plates were washed with PBS and incubated with $100 \mu \mathrm{L}$ of TMB solution for 20 minutes at room temperature. Staining was stopped using stop solution and absorbance at $490 \mathrm{~nm}$ was 
measured. EGF levels were expressed as picograms per milligram of total protein.

\section{Statistical analysis}

The statistical analysis was performed using IBM SPSS ver. 22.0 (IBM Corp., Armonk, NY, USA). Differences in the percentage area of skin defects between the experimental and control groups were analyzed using the paired t-test. The mean capillary density and ELISA results for EGF were also analyzed using the paired t-test. P-values $<0.05$ were considered to indicate statistical significance.

\section{Fig. 2. A line graph of results from the mouse model}

An accelerated decrease of wound size was observed on days four, seven, and 10 in the experimental group, which was found to be a statistically significant difference $\left({ }^{*} \mathrm{P}<0.001\right)$.

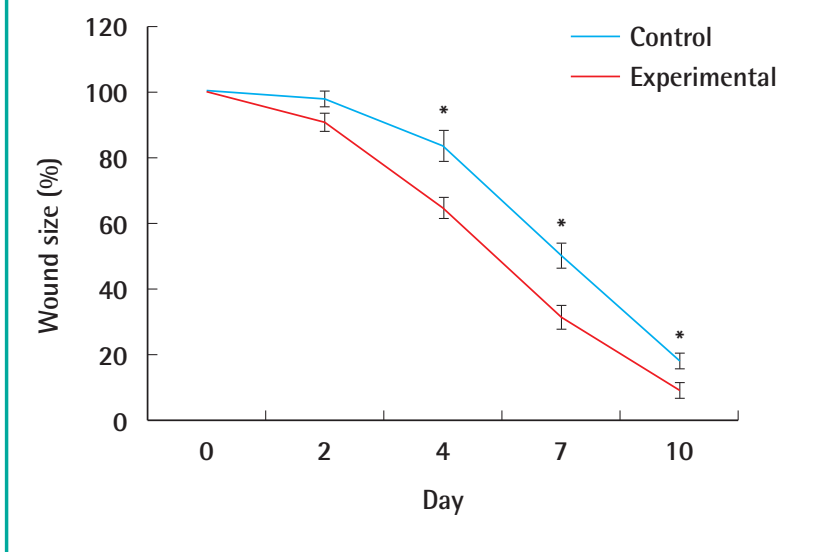

\section{RESULTS}

\section{Wound size}

Rose placenta accelerated wound healing with statistical significance in the experimental group (Fig. 2). On day 2, the mean wound size was $97.73 \%$ in the control group and $90.89 \%$ in the experimental group. The decrease in size was more remarkable in the experimental group, but the results were not statistically significant $(\mathrm{P}=0.863)$. On days 4,7 , and 10 , the mean wound size was $83.70 \%, 49.73 \%$, and $17.70 \%$ in the control group, respectively, compared to $64.72 \%, 30.89 \%$, and $8.72 \%$ in the experimental group, respectively. We observed faster wound healing in the experimental group $(\mathrm{P}<0.001)$. No complications, such as wound infection or fluid collection, occurred.

\section{Histopathologic features}

We observed more inflammation in the control group on day 2. Neutrophil counts were significantly higher in the control group (mean, 792.55 cells $/ \mathrm{mm}^{2}$ ) than in the experimental group (mean, 511.68 cells $\left./ \mathrm{mm}^{2}\right)(\mathrm{P}=0.001)$ (Table 1$)$. On days 4,7 , and 10 , no significant differences were observed between the groups with regard to the number of neutrophils. On day 10,

\section{Table 1. Mean inflammatory cell count on day 2}

\begin{tabular}{|lcc|}
\hline Cell count & Control group & Experimental group \\
\hline Mean cell count per field & $28.5 \pm 6.19$ & $18.4 \pm 3.06$ \\
Mean cell count per 1 $\mathrm{mm}^{2}$ & $792.55 \pm 172.04$ & $511.68 \pm 85.15$ \\
P-value & \multicolumn{2}{|c|}{0.001} \\
\hline a) & \multicolumn{2}{|c}{-test. } \\
\end{tabular}

\section{Fig. 3. Histologic findings $(H \& E, \times 400)$}

On day 10, a more mature dermal structure was observed in the experimental group than in the control group. (A) Control group. (B) Experimental group.
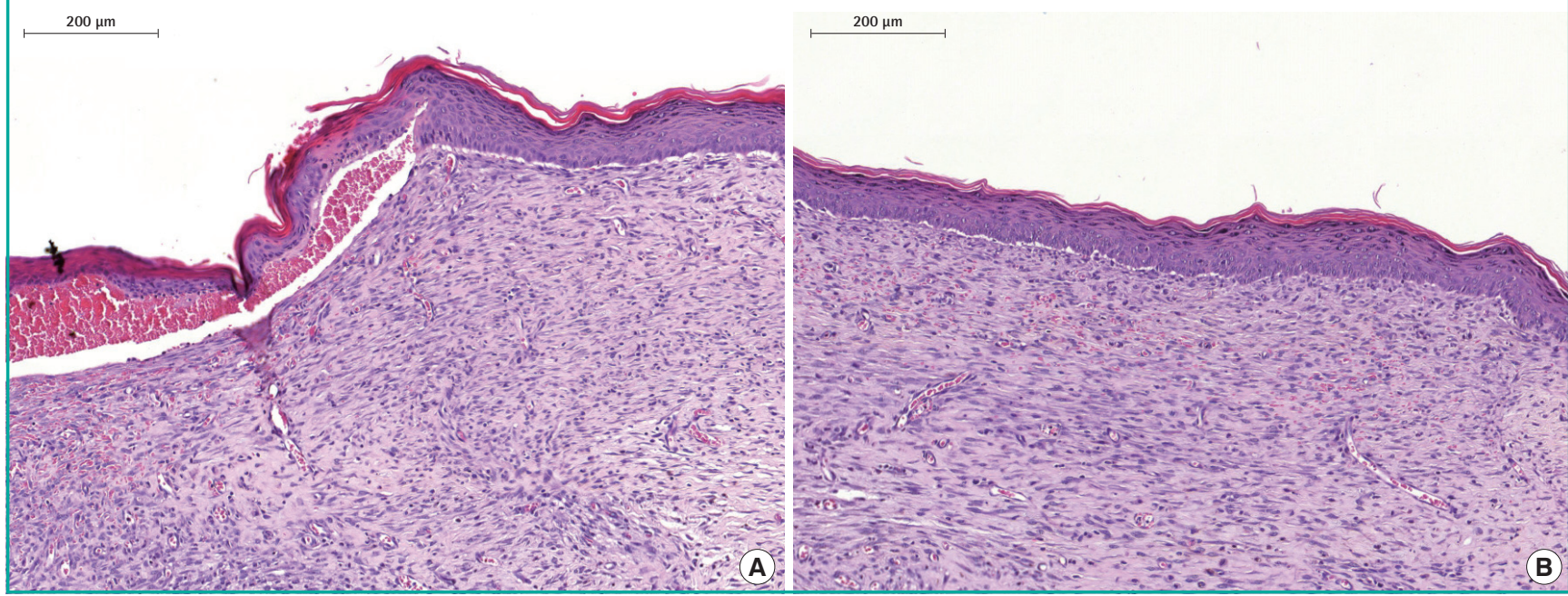


\section{Fig. 4. Immunohistochemical findings of VEGF and EGF $(\times 200)$}

Immunohistochemical findings of VEGF and EGF in mouse tissue on day 2. VEGF was expressed in experimental group more than in the control group. EGF was expressed in the experimental group more than in the control group. (A) VEGF in the control group, (B) VEGF in the experimental group, (C) EGF in the control group, (D) EGF in the experimental group. VEGF, vascular endothelial growth factor; EGF, epidermal growth factor.
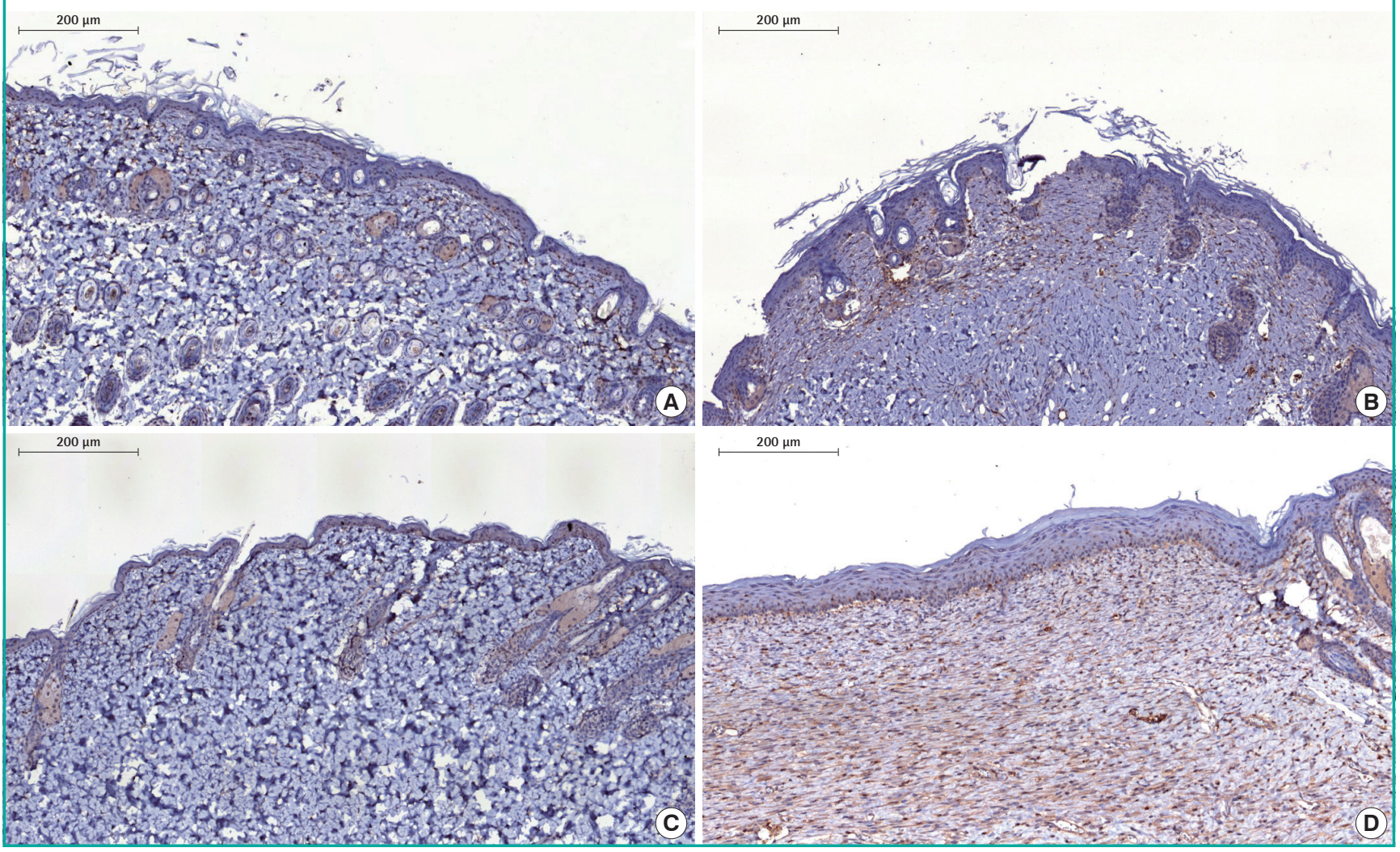

Table 2. Mean vessel density on day 10

\begin{tabular}{|lcc|}
\hline Cell count & Control group & Experimental group \\
\hline Mean cell count per field & $10.4 \pm 1.95$ & $14.8 \pm 2.25$ \\
Mean cell count per $1 \mathrm{~mm}^{2}$ & $70.35 \pm 13.24$ & $100.12 \pm 15.22$ \\
P-value & & \\
\hline a) & & 0.001 \\
\hline
\end{tabular}

parakeratosis and erythrocyte extravasation were observed, and the dermal structure of the experimental group was confirmed to be more mature than that of the control group (Fig. 3).

\section{Expression of VEGF, EGF, and TGF- $\beta 1$}

We observed increased levels of VEGF and EGF expression in the experimental group compared to the control group on day 2 (Fig. 4). On days 4, 7, and 10, we observed minimal expression of VEGF and EGF in both groups, and no significant differences were observed in the extent of expression. The expression of TGF- $\beta 1$ was greater in the control group than in the experimental group on days 7 and 10, but not on days 2 and 4 (Fig. 5).

Therefore, we conclude that the expression of VEGF and EGF at an early phase increased in the experimental group, while the expression of TGF- $\beta 1$ at a later phase decreased in the experimental group.

\section{Vessel density}

Vessel density was measured by CD31 positivity. We did not observe a statistically significant difference in mean vessel density between the groups on day $4(\mathrm{P}=0.721)$. However, we confirmed a higher mean vessel density in the experimental group than in the control group on day 10 (Table 2, Fig. 6). This difference was found to be statistically significant $(\mathrm{P}=0.001)$.

\section{Quantitative analysis of EGF expression with ELISA}

We confirmed that the expression of EGF was statistically twofold higher in the experimental group on day $2(\mathrm{P}=0.001)$. The expression of EGF tended to decrease over time. On days 4, 7, and 10, a higher expression of EGF was seen in the experimental group than in the control group. However, this result was not statistically significant (Fig. 7). 


\section{Fig. 5. Immunohistochemical findings of TGF- $\beta(\times 200)$}

On day four, no difference was found in the extent of expression between the control group and the experimental group. On day 10, TGF- $\beta 1$ was expressed in the control group more than in the experimental group. (A) Control group on day four, (B) experimental group on day four, (C) control group on day 10, (D) experimental group on day 10. TGF- $\beta 1$, transforming growth factor- $\beta 1$.
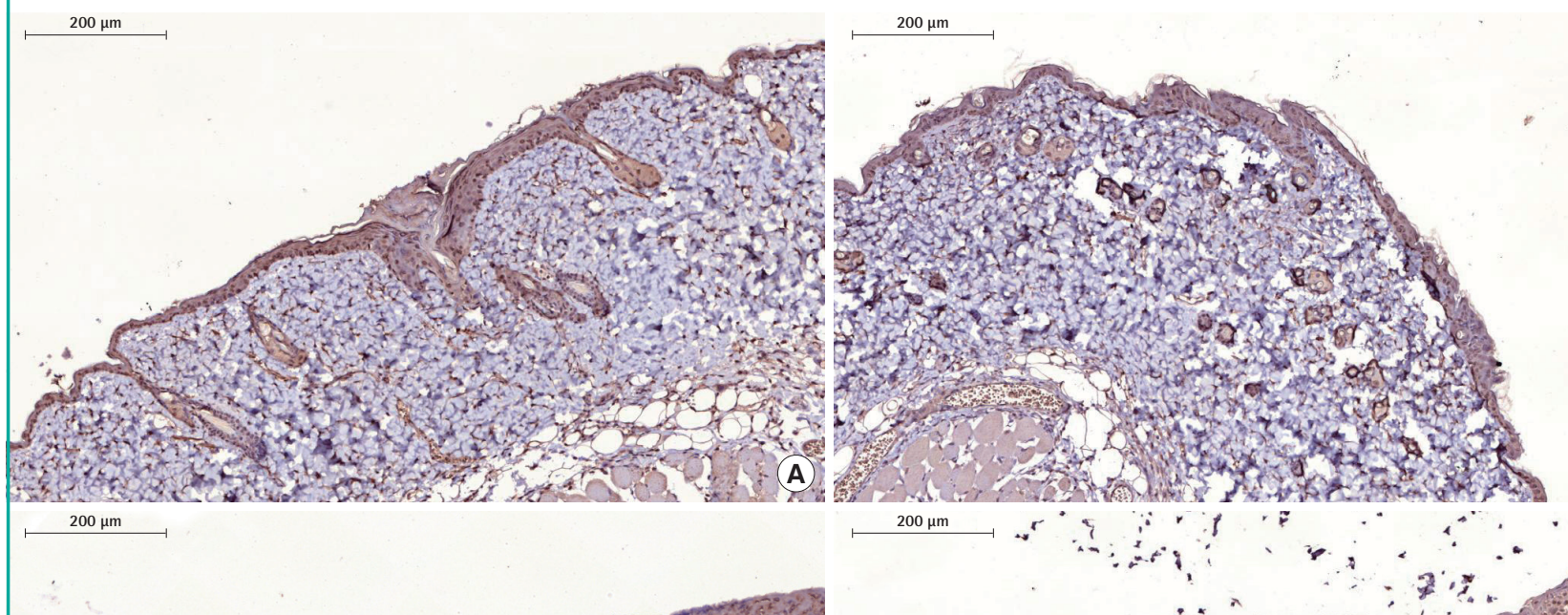
$200 \mu \mathrm{m}$
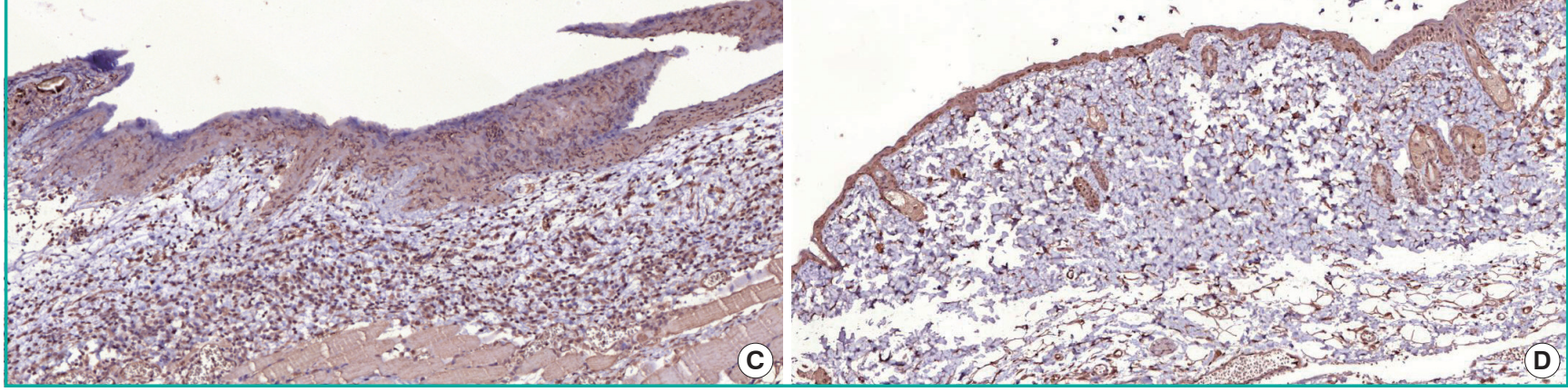

\section{Fig. 6. Immunohistochemical findings of CD31 positivity $(\times 200)$}

On day 10, the mean vessel density was higher in the experimental group than in the control group. A statistically significant difference was found $(\mathrm{P}<0.05)$. (A) Control group, (B) experimental group.
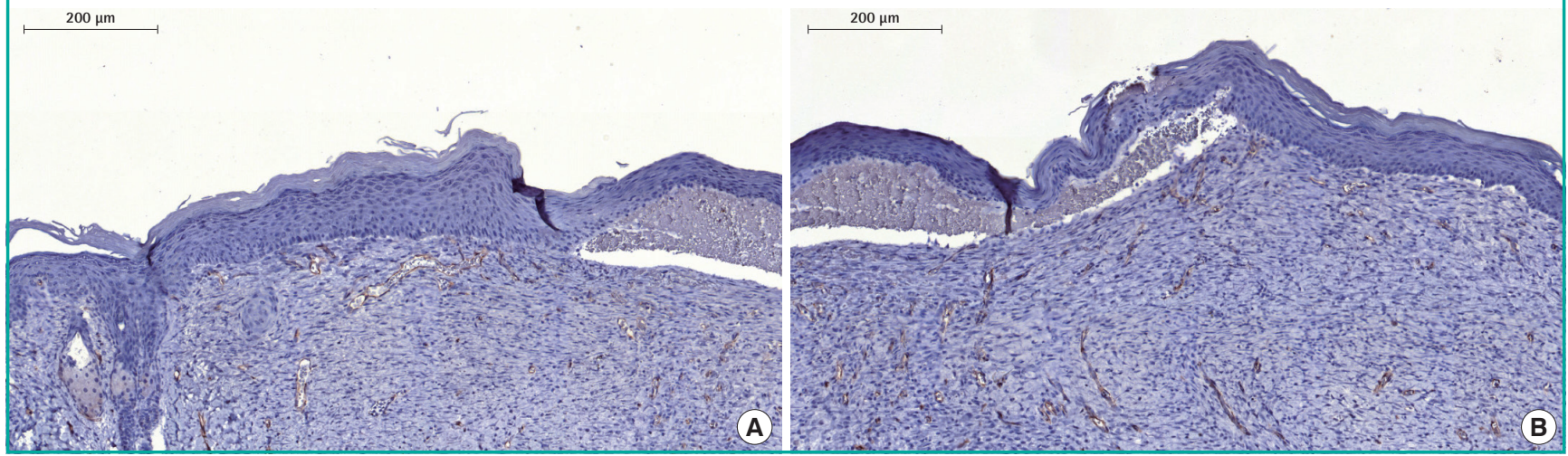

\section{DISCUSSION}

It is well known that a range of components in $R$. damascena, including $\beta$-citronellol, nonadecane, geraniol, nerol, and kaempferol [3,11-13], contribute to its medical effectiveness.

Few reports have investigated wound healing and $R$. damascena. Mouthwash containing $R$. damascena showed treatment effects on recurrent aphthous stomatitis [14]. In an animal study of New Zealand rabbit ear wounds, alcoholic fractions of $R$. damascena accelerated the epithelialization of the wounds [15].

Many plants promote the wound healing mechanism and therefore have a tremendous potential for therapeutic use in wound care. Studies have suggested that the use of herbal medicines may accelerate the wound healing process by promoting 


\section{Fig. 7. EGF concentration on days two, four, seven, and 10}

Note that the concentration of EGF was twofold higher in the experimental group on day two $\left({ }^{*} P=0.001\right)$. On days four, seven, and 10 , a slight increase in the expression of EGF was found in the experimental group compared to the control group. However, the difference was not significant. EGF, epidermal growth factor; POD, post operative day.

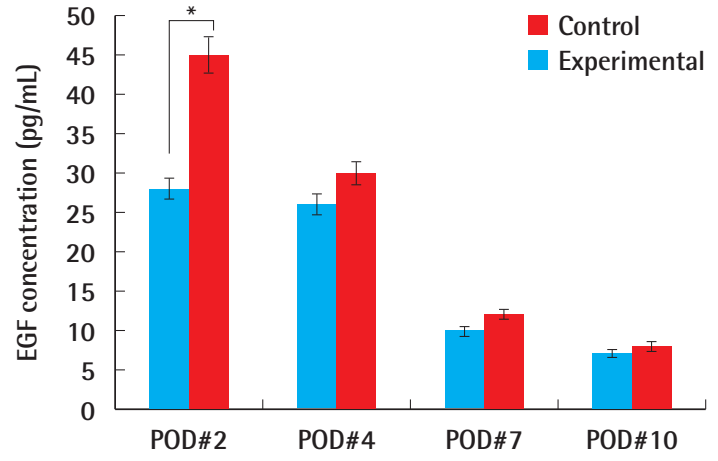

growth factor release. For example, grape seed extract triggers the release of VEGF [16]. R. damascena is also involved in the enhancement of neurite outgrowth [17]. A higher level of EGF was observed in the experimental group in our study, but the relationship between $R$. damascena and EGF remains to be elucidated.

Wound healing is a complex process that requires the collaboration of many tissues and cells. Many different cytokines also participate in this process, and the predominant cells or cytokines change during the various phases of wound healing. However, it is not easy to investigate wound healing and associated factors because in vitro tests are not always reproducible in vivo. EGF is known to stimulate wound healing. It promotes wound healing by increasing the rate of epidermal proliferation and accelerating the level of wound contraction related to myofibroblast proliferation and collagen deposition [18]. It is also essential in the regulation of differentiation, proliferation, and survival of cells.

Prior to this research, we conducted several pilot studies showing increased EGF levels. Other studies have addressed the efficacy of EGF on wound healing [19]; as such, EGF is one of the key growth factors associated with wound healing.

In our experiments, two 8-mm excisional wounds were made on the back of each mouse with a dermal biopsy punch, and the wounds were covered with Tegaderm (3M Health Care, Maplewood, MN, USA) to keep them moist [20]. The size of the wounds decreased rapidly in the experimental group on days 2 , 4,7 , and 10. The largest size difference was seen on days 4 and 7 , and increases in the expression of EGF were seen starting on day 2. Higher expression of EGF was confirmed in the experimental group on day 2 by immunohistochemistry and ELISA.

EGF is known to play a key role during the proliferation phase.
However, we encountered increased levels in the early phase of our experiment, which is thought to be the inflammatory phase. Guo and Dipietro [21] demonstrated that EGF can promote the inflammatory phase and function as a chemoattractant. Repertinger et al. [22] showed that EGF regulates multiple facets of cutaneous wound healing, including inflammation, wound contraction, proliferation, migration, and angiogenesis. It seems that EGF is able to accelerate the total process of wound healing by controlling the early phase of wound healing. We can therefore conclude that the decreased wound size in the experimental group on days 4 and 7 was due to an increased EGF level in the early phase, which led to the acceleration of the wound healing process.

On days 4, 7, and 10, a slight increase in EGF expression was seen in the experimental group, although this result was statistically insignificant. Tanaka et al. [23] showed that the continuous presence of EGF is necessary for maximum wound healing, and demonstrated that solutions of EGF drain quickly. We injected rose placenta once in the beginning of the experiment. The absence of a significant difference in the levels of EGF between the experimental and control group on those days might have been due to the fast drainage of the rose placenta extract.

No differences in the expression of TGF- $\beta 1$ in the early phase were observed, but a higher level of expression was seen in the control group in the late phase, suggesting that more inflammation and scar formation were present in the control group [24]. Beanes et al. [25] found that TGF- $\beta 1$ promoted inflammatory cell infiltration and scarring. If this is the case for scars, rose placenta might also be effective in scar remodeling.

Prominent angiogenesis in the experimental group compared to the control group was also observed with CD31+ immunohistochemistry. Vessel density was determined by counting capillaries with small diameters and walls that consist of only a single layer of endothelium. The number of newly formed vessels was greater in the experimental group on day 10, but no differences were found on day 4 . In contrast, higher VEGF expression was seen at the early phase on days 2 and 4 . The process of angiogenesis requires a specific period of time after VEGF expression, which might explain the results described above.

This study had some limitations. The specific components that supposedly promote wound healing were not evaluated. A single component or combination thereof, such as $\beta$-citronellol, nonadecane, geraniol, nerol, and kaempferol may be responsible for the effects $[3,11-13]$. Further research is necessary to elucidate this issue.

Substances with unproven medical effects are usually provided as topical agents to minimize side effects. In order to stabilize their medical effect on the wound site, topical agents are usually 
prepared with ointment base. We have encountered difficulties in finding an appropriate oint basement to enable the delivery of extracts in a topical form, which is why rose placenta extract was administered as an injection. If possible, in future studies it would be better to apply rose placenta as a topical agent.

In addition, although we obtained successful results in mice, it is not known whether the same results would be obtained in humans. In mice, no significant side effects of rose placenta extract were observed, however, it is possible that some side effects may be found in humans. Additionally, neither the effective capacity nor usage in humans has yet been studied.

Rose placenta is a natural substance that can be obtained easily and inexpensively. Unlike synthetic growth factors, it can be extracted from a natural product and used as a stable wound healing agent. In conclusion, rose placenta was found to promote wound healing in a murine full-thickness wound model by increasing EGF release. Rose placenta may be an excellent candidate for drug development to accelerate wound healing

\section{REFERENCES}

1. Howell-Jones RS, Wilson MJ, Hill KE, et al. A review of the microbiology, antibiotic usage and resistance in chronic skin wounds. J Antimicrob Chemother 2005;55:143-9.

2. Slavov A, Kiyohara H, Yamada H. Immunomodulating pectic polysaccharides from waste rose petals of Rosa damascena Mill. Int J Biol Macromol 2013;59:192-200.

3. Boskabady MH, Shafei MN, Saberi Z, et al. Pharmacological effects of rosa damascena. Iran J Basic Med Sci 2011;14: 295-307.

4. Teoh SL, Latiff AA, Das S. The effect of topical extract of Momordica charantia (bitter gourd) on wound healing in nondiabetic rats and in rats with diabetes induced by streptozotocin. Clin Exp Dermatol 2009;34:815-22.

5. Bani S, Hasanpour S, Mousavi Z, et al. The effect of rosa damascena extract on primary dysmenorrhea: a double-blind cross-over clinical trial. Iran Red Crescent Med J 2014;16: e14643.

6. Lynch SE, Colvin RB, Antoniades HN. Growth factors in wound healing: single and synergistic effects on partial thickness porcine skin wounds. J Clin Invest 1989;84:640-6.

7. Greenhalgh DG, Sprugel KH, Murray MJ, et al. PDGF and FGF stimulate wound healing in the genetically diabetic mouse. Am J Pathol 1990;136:1235-46.

8. Schultz GS, White M, Mitchell R, et al. Epithelial wound healing enhanced by transforming growth factor-alpha and vaccinia growth factor. Science 1987;235:350-2.

9. Shirakata Y, Kimura R, Nanba D, et al. Heparin-binding
EGF-like growth factor accelerates keratinocyte migration and skin wound healing.J Cell Sci 2005;118:2363-70.

10. Nissen NN, Polverini PJ, Koch AE, et al. Vascular endothelial growth factor mediates angiogenic activity during the proliferative phase of wound healing. Am J Pathol 1998;152: 1445-52.

11. Loghmani-Khouzani H, Fini OS, Safari J. Essential oil composition of Rosa damascena Mill cultivated in central Iran. Sci Iranica 2007;14:316-9.

12. Ulusoy S, Bosgelmez-Tinaz G, Secilmis-Canbay H. Tocopherol, carotene, phenolic contents and antibacterial properties of rose essential oil, hydrosol and absolute. Curr Microbiol 2009;59:554-8.

13. Aydinli M, Tutaş M. Production of rose absolute from rose concrete. Flavour Fragr J 2003; 18:26-31.

14. Hoseinpour H, Peel SA, Rakhshandeh H, et al. Evaluation of Rosa damascena mouthwash in the treatment of recurrent aphthous stomatitis: a randomized, double-blinded, placebo-controlled clinical trial. Quintessence Int 2011;42: 483-91.

15. Mahmoudian A, Rakhshandeh H, Khayatzadeh J, et al. Study of the healing effects of alcoholic fractions of Rosa Damascena on the epithelialization process of the New Zealand rabbit's ear wounds. Pharmaologyonline 2009;3:130-5.

16. Hemmati AA, Foroozan M, Houshmand G, et al. The topical effect of grape seed extract $2 \%$ cream on surgery wound healing. Glob J Health Sci 2015;7:52-8.

17. Jin S, Zhang M, Gao Y, et al. The efficacy of Jing Wan Hong ointment for nerve injury diabetic foot ulcer and its mechanisms. J Diabetes Res 2014;2014:259412.

18. Kwon YB, Kim HW, Roh DH, et al. Topical application of epidermal growth factor accelerates wound healing by myofibroblast proliferation and collagen synthesis in rat.J Vet Sci 2006;7:105-9.

19. Brown GL, Nanney LB, Griffen J, et al. Enhancement of wound healing by topical treatment with epidermal growth factor. N Engl J Med 1989;321:76-9.

20. Mirza R, Koh TJ. Dysregulation of monocyte/macrophage phenotype in wounds of diabetic mice. Cytokine 2011;56: 256-64.

21. Guo S, Dipietro LA. Factors affecting wound healing.J Dent Res 2010;89:219-29.

22. Repertinger SK, Campagnaro E, Fuhrman J, et al. EGFR enhances early healing after cutaneous incisional wounding. J Invest Dermatol 2004;123:982-9.

23. Tanaka A, Nagate T, Matsuda H. Acceleration of wound healing by gelatin film dressings with epidermal growth factor. J Vet Med Sci 2005;67:909-13. 
24. Hou Q He WJ, Hao HJ, et al. The four-herb Chinese medicine ANBP enhances wound healing and inhibits scar formation via bidirectional regulation of transformation growth factor pathway. PLoS One 2014;9:e112274.
25. Beanes SR, Dang C, Soo C, et al. Skin repair and scar formation: the central role of TGF-beta. Expert Rev Mol Med 2003;5:1-22. 